\title{
Mixed Generalized Multiscale Finite Element Method for a Simplified Magnetohydrodynamics Problem in Perforated Domains
}

\author{
Valentin Alekseev ${ }^{1}$, Qili Tang ${ }^{2}$, Maria Vasilyeva ${ }^{1,3}$, Eric T. Chung ${ }^{4}$ and Yalchin Efendiev ${ }^{5, *}$ \\ 1 Multiscale Model Reduction Laboratory, North-Eastern Federal University, 677007 Yakutsk, Russia; \\ alekseev.valen@mail.ru (V.A.); vasilyevadotmdotv@gmail.com (M.V.) \\ 2 Hunan Key Laboratory for Computation and Simulation in Science and Engineering, Key Laboratory of \\ Intelligent Computing \& Information Processing of Ministry of Education, School of Mathematics and \\ Computational Science, Xiangtan University, Xiangtan 411105, China; tangqili@xtu.edu.cn \\ 3 Institute for Scientific Computation, Texas A\&M University, College Station, TX 77843, USA \\ 4 Department of Mathematics, The Chinese University of Hong Kong, Shatin, New Territories, \\ Hong Kong 999077, China; eric.t.chung@gmail.com \\ 5 Department of Mathematics, Texas A\&M University, College Station, TX 77843, USA \\ * Correspondence: efendiev@math.tamu.edu
}

Received: 22 May 2020; Accepted: 17 June 2020; Published: 23 June 2020

\begin{abstract}
In this paper, we consider a coupled system of equations that describes simplified magnetohydrodynamics (MHD) problem in perforated domains. We construct a fine grid that resolves the perforations on the grid level in order to use a traditional approximation. For the solution on the fine grid, we construct approximation using the mixed finite element method. To reduce the size of the fine grid system, we will develop a Mixed Generalized Multiscale Finite Element Method (Mixed GMsFEM). The method differs from existing approaches and requires some modifications to represent the flow and magnetic fields. Numerical results are presented for a two-dimensional model problem in perforated domains. This model problem is a special case for the general 3D problem. We study the influence of the number of multiscale basis functions on the accuracy of the method and show that the proposed method provides a good accuracy with few basis functions.
\end{abstract}

Keywords: generalized multiscale finite element method; magnetohydrodynamics; perforated domain

\section{Introduction}

The magnetohydrodynamic (MHD) system describes the interactions of electrically conducting incompressible flows in the presence of a magnetic field. MHD flows are usually involved in the contexts: liquid metal cooling of nuclear reactors, electromagnetic casting of metals, MHD generators, accelerators, and MHD ion propulsion, see [1-3]. The equations that model the MHD are the coupling of incompressible Navier-Stokes equations and the Maxwell's equations via the Lorentz force and Ohm's law. There are some research works devoted to the numerical approximation of MHD equations. For treating the nonlinear terms effectively, three classical iterative methods (Stokes-type, Newtown and Oseen-type ones) within finite element approximation for the steady MHD equations have been developed in $[4,5]$. Especially, the theoretical analysis and numerical examples in $[4,6]$ show that the Picard iteration (called Oseen-type iteration by the authors in the reference) is suitable for high Reynolds number problems. The local and parallel finite element algorithms based on two-grid methods were given in [7-9]. The stabilized finite element schemes that capture physical solutions were investigated in [10]. The mixed finite element methods with divergence-free velocities and magnetics fields are developed [11-13]. However, analysis of the above literature mainly focuses on 
the singly-connected computational domains. In this paper, our goal is to develop multiscale methods for MHD flows on complex domains with perforations.

In this paper, we consider a simplified MHD problem in perforated domains, and propose a multiscale method for constructing the reduced order model on the coarse grid. In particular, we consider a special 2D case of MHD equations and the full MHD problem will be considered in the future. We start with the mixed formulation for both equations of the simplified MHD problem in a perforated domain. For the solution of the nonlinear system, we use a Picard iteration to decouple the equations. We construct a fine grid that resolves perforations on the grid level and write an approximation using a mixed finite element method. Problems in perforated domains have a multiscale nature and approximations on the fine grid lead the large system of equations. To reduce the size of the discrete system, multiscale methods or homogenization techniques are needed [14-19]. Homogenization techniques are constructed to solve problems with scale separation, i.e., when perforations are of similar sizes. When the perforated media do not have scale separation, multiscale methods are used [20-24]. In the papers [20,21], the authors presented the multiscale finite element method for problems in perforated domains, but these approaches use a limited number of degrees of freedom per coarse element. In the papers [18,25], the general approach for constructing the multiscale basis functions using local spectral characteristics was presented in $[18,25]$. Continuous and Discontinuous Galerkin Generalized Multiscale Finite Element Methods (GMsFEM) for coarse grid approximation of the problems in perforated domains was presented in $[26,27]$. The GMsFEM is a systematic approach to identify multiscale basis functions via local snapshots and local spectral problems. The local snapshots are constructed by solving local problems and contain the information about local geometry structure (perforations). By performing local spectral decomposition, the method identifies multiscale basis functions. For accurately approximating the fluxes, we use a Mixed Generalized Multiscale Finite Element method and construct multiscale basis functions for them [28-31]. In Mixed GMsFEM, we construct the snapshot space for each coarse edge, perform the local spectral decomposition in the snapshot space and select the dominant modes as multiscale basis functions for the flux. The presented work is based on our previous papers [30,31], where we presented a Mixed GMsFEM for solutions of the problems in perforated domains. In this paper, we apply and study the method for solving the incompressible magnetohydrodynamics (MHD) problem in perforated domains. We present numerical results for some perforated geometries 2D. These numerical results demonstrate one can achieve a good accuracy with fewer basis functions.

The paper is organized as follows. In Section 2, we describe the problem formulation. Next, we decouple the system of equations using Picard iterations. In Sections 3 and 4 , we present a fine grid and multiscale approximations for the magnetic field and flow problems, respectively. Fine grid and coarse grid approximations are presented using mixed formulation. Construction of the multiscale basis functions are presented. In Section 5, we present numerical results for the two-dimensional problem in a perforated domain. The paper ends with a conclusion.

\section{Problem Formulation}

The full steady incompressible MHD equations in $\Omega \subset R^{3}$ is a complex system which link the velocity field $u=\left(u_{1}, u_{2}, u_{3}\right)$, magnetic field $B=\left(B_{1}, B_{2}, B_{3}\right)$ and pressure $p$ :

$$
\begin{array}{rlrl}
-R_{e}^{-1} \Delta u+u \cdot \nabla u+\nabla p-S_{c} \operatorname{curl} B \times B & =f, & & x \in \Omega, \\
R_{e_{m}}^{-1} \operatorname{curl}(\operatorname{curl} B)-\operatorname{curl}(u \times B) & =g, & & x \in \Omega, \\
\nabla \cdot u=0, & x \in \Omega, \\
\nabla \cdot B=0, & x \in \Omega,
\end{array}
$$

where $R_{e}$ is the hydrodynamic Reynolds number, $R_{e_{m}}$ the magnetic Reynolds number, $S_{c}$ the coupling number. We refer the readers to [32] for details on the existence, uniqueness, and finite element approximation of solutions of (1). 
This article considers a magnetic field $B=(0,0, B)$ perpendicular to the velocity field $u=$ $\left(u_{1}, u_{2}, 0\right)$ of $(1)$ in two-dimensional perforated domain $\Omega$, then the simplified MHD equations without any body force and hydrodynamic convective terms are described as follows:

$$
\begin{aligned}
\nabla \cdot(u B)-\nabla \cdot\left(R_{e_{m}}^{-1} \nabla B\right) & =0, & & x \in \Omega, \\
-R_{e}^{-1} \Delta u+\nabla p-S_{c} B \nabla B & =0, & & x \in \Omega, \\
\nabla \cdot u & =0, & & x \in \Omega .
\end{aligned}
$$

Let $D=R_{e_{m}}^{-1}, q=-D \nabla B$ be the flux and $\Omega$ is the perforated domain (see Figure 1). We write Equation (2) in the mixed form

$$
\begin{aligned}
D^{-1} q+\nabla B=0, & x \in \Omega, \\
\nabla \cdot q+\nabla \cdot(u B)=0, & x \in \Omega, \\
-R_{e}^{-1} \Delta u+\nabla p+S_{c} D^{-1} B q=0, & x \in \Omega, \\
\nabla \cdot u=0, & x \in \Omega,
\end{aligned}
$$

and consider with the following boundary conditions

$$
\begin{gathered}
u=0, \quad x \in \Gamma_{P}, \quad(\nabla u-p \mathcal{I}) \cdot n=0, \quad x \in \partial \Omega / \Gamma_{P}, \\
B=0, \quad x \in \Gamma_{P}, \quad B=g, \quad x \in \Gamma_{1}, \quad q \cdot n=0, \quad x \in \Gamma_{2},
\end{gathered}
$$

where $\Gamma_{P}$ is the boundary of perforations, $\Gamma_{1} \cup \Gamma_{2} \cup \Gamma_{P}=\partial \Omega, n$ is the unit outward normal vector on $\partial \Omega$ and $\mathcal{I}$ is the $n \times n$ identity matrix.

$\Omega$

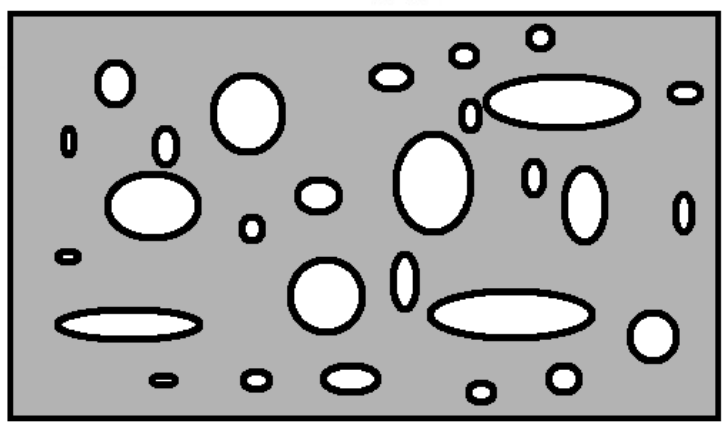

Figure 1. Illustration of a perforated domain.

For linearization, we use Picard iterations and obtain the following algorithm

- $\quad$ Find $\left(q^{k+1}, B^{n+1}\right)$ from

$$
\begin{aligned}
D^{-1} q^{k+1}+\nabla B^{k+1} & =0, \\
\nabla q^{k+1}+\nabla\left(u^{k} B^{k+1}\right) & =0,
\end{aligned}
$$

- $\quad$ Find $\left(u^{k+1}, p^{n+1}\right)$ from

$$
\begin{aligned}
-R_{e}^{-1} \Delta u^{k+1}+\nabla S_{c} p^{k+1}+S_{c} D^{-1} B^{k+1} q^{k+1} & =0, \\
\nabla u^{k+1} & =0 .
\end{aligned}
$$

Here $k$ is the nonlinear iteration. 


\section{Magnetic Field Problem}

We have the following variational formulations: find $\left(q^{k+1}, B^{n+1}\right) \in V^{q} \times Q^{B}$ such that

$$
\begin{gathered}
m\left(q^{k+1}, v\right)+g\left(B^{k+1}, v\right)=f_{q}(v) \quad \forall v \in V^{q}, \\
g\left(q^{k+1}, r\right)+c\left(B^{k+1}, r\right)=0, \quad \forall r \in Q^{B},
\end{gathered}
$$

where $k$ is the nonlinear iteration and

$$
\begin{gathered}
m(q, v)=-\int_{\Omega} D^{-1} q \cdot v d x, \quad g(B, q)=\int_{\Omega} B \nabla q d x, \\
c(B, r)=\int_{\Omega}\left(u^{k} \nabla B\right) \cdot r d x, \quad f_{q}(v)=\int_{\Gamma_{1}} g v \cdot n d s .
\end{gathered}
$$

We can rewrite fine grid approximation in the matrix form

$$
\left(\begin{array}{cc}
M & G^{T} \\
G & C^{k}
\end{array}\right)\left(\begin{array}{l}
q^{k+1} \\
B^{k+1}
\end{array}\right)=\left(\begin{array}{c}
F_{q} \\
0
\end{array}\right)
$$

where $C^{k}=C\left(u^{k}\right)$. The fine grid problem is solved using the lowest-order Raviart-Thomas element. Multiscale Approximation

To construct the multiscale space for the coarse scale approximation, we use a Mixed GMsFEM [23,28-31]. Let $\mathcal{T}^{H}$ be a coarse grid of domain $\Omega$ and $\mathcal{E}^{H}$ be the set of all faces of the coarse grid, where $H$ is the coarse mesh size and $N_{e}$ be the total number of faces. We define the neighborhood of the face $E_{i} \in \mathcal{E}^{H}$ by

$$
\omega_{i}=\bigcup_{j}\left\{K_{j} \in \mathcal{T}^{H} \mid E_{i} \in \partial K_{j}\right\} .
$$

and $\omega_{i}$ is a union of two coarse grid blocks when $E_{i}$ lies in the interior of the domain $\Omega$ (see Figure 2).

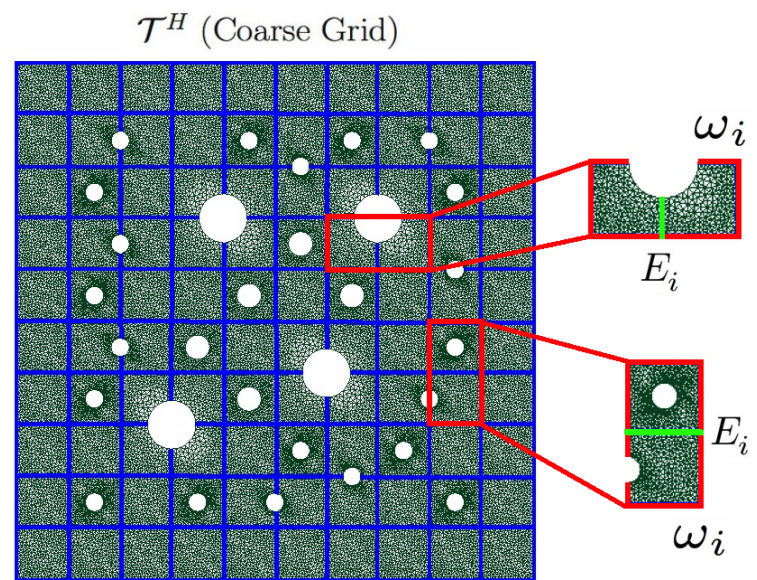

Figure 2. Illustration of a coarse grid and local domain $\left(\omega_{i}\right)$ for the Magnetic field problem.

We construct a multiscale space for the flux

$$
V^{q, H}=\operatorname{span}\left\{\psi_{i}\right\}_{i=1}^{N_{q}}, \quad Q^{B, H}=\left\{r \in L^{2}(\Omega):\left.r\right|_{K} \in P^{0}(K), \forall K \in \mathcal{T}_{H}\right\}
$$

and for the $B$, we use the space of piecewise constant functions over the coarse triangulation $\mathcal{T}^{H}$. 
Here $\psi_{i}$ are the multiscale basis functions that supported in a coarse neighborhood $\omega_{i}, N_{q}=\operatorname{dim}\left(V^{q, H}\right)$ is the number of basis functions and $N_{B}=\operatorname{dim}\left(Q^{B, H}\right)$ is equal to the number of coarse grid cells .

For the coarse grid approximation, we use a Mixed GMsFEM and have the following variational formulation: find $\left(q_{H}^{k+1}, B_{H}^{k+1}\right) \in V^{q, H} \times Q^{B, H}$ such that

$$
\begin{gathered}
m\left(q_{H}^{k+1}, v\right)+g\left(B_{H}^{k+1}, v\right)=f(v) \quad \forall v \in V^{q, H} \\
g\left(q_{H}^{k+1}, r\right)+c\left(B_{H}^{k+1}, r\right)=0, \quad \forall r \in Q^{B, H}
\end{gathered}
$$

We can write our system in matrix form for each nonlinear iteration

$$
\left(\begin{array}{cc}
M_{H} & G_{H} \\
G_{H}^{T} & C_{H}
\end{array}\right)\left(\begin{array}{c}
q_{H} \\
B_{H}
\end{array}\right)=\left(\begin{array}{c}
F_{H} \\
0
\end{array}\right)
$$

where

$$
M_{H}=R_{q} M R_{q}^{T}, \quad G_{H}=R_{q} G R_{B}^{T}, \quad C_{H}=R_{B} C R_{B}^{T}, \quad F_{H}=R_{q} F_{q} .
$$

Here $R_{q}$ and $R_{B}$ are the projection matrices

$$
R_{q}=\left[\psi_{1}, \ldots, \psi_{N_{q}}\right], \quad R_{B}=\left[\eta_{1}, \ldots, \eta_{N_{B}}\right]
$$

We remark that $\left\{\psi_{i}\right\}_{i=1}^{N_{q}}$ are the multiscale basis functions for flux and $\left\{\eta_{i}\right\}_{i=1}^{N_{B}}$ are the basis functions for $B$.

For construction of the multiscale space for the flux, we start with the construction of the snapshot space that contains an extensive set of basis functions formed by the solution of local problems with all possible boundary conditions up to the fine grid resolution. After that, we solve a spectral problem to select dominant modes in the snapshot space.

Snapshot space. We solve the following problem on the coarse neighborhood $\omega_{i}$ that corresponds to the coarse-grid edge $E_{i} \in \mathcal{E}^{H}$ : Find $\left(\phi_{l}^{i}, \eta\right)$ such that

$$
\begin{gathered}
m\left(\phi_{l}^{i} v\right)+g(\eta, v)=0, \quad v \in V_{h^{\prime}}^{i} \\
g\left(\phi_{l}^{i}, r\right)=\int_{\omega_{i}} c r d x, \quad r \in Q_{h^{\prime}}^{i}
\end{gathered}
$$

with boundary condition $\phi_{l}^{i} \cdot n_{i}=0$ on $\partial \omega_{i}$ and additional boundary condition $\phi_{l}^{i} \cdot n_{i}=\delta_{l}^{i}$ on coarse edge $E_{i}$, where $n_{i}$ is the outward unit-normal vector on $\partial \omega_{i}$. The local problem (10) is solved separately on each coarse-grid element $K_{j}^{i} \subset \omega_{i}$ using the fine-grid defined in $\omega_{i}$ by the lowest-order Raviart-Thomas element. Here $E_{i}=\cup_{l=1}^{J_{i}} e_{l}$, where $e_{l}$ are the fine-grid edges on $E_{i} / \Gamma_{P}$, and $J_{i}$ is the number of the fine grid edges on $E_{i} / \Gamma_{P}$. The delta function $\delta_{l}^{i}$ is a piecewise constant function defined on $E_{i} / \Gamma_{P}$ such that it has value 1 on $e_{l}$ and value 0 on the other fine-grid edges. The constant $c$ in (10) is chosen by compatibility condition, $c=\frac{1}{\left|K_{j}^{i}\right|} \int_{E_{i}} \phi_{l}^{i} \cdot n_{i} d s, j=1,2$.

The collection of the solutions of the above local problems generates the snapshot space in $\omega_{i}$

$$
V^{i, \text { snap }}=\left\{\phi_{l}^{i}: 1 \leq l \leq J_{i}\right\}, \quad R_{i, \text { snap }}=\left[\phi_{1}^{i}, \ldots, \phi_{J_{i}}^{i}\right]
$$

Multiscale space. For reduction on the snapshot space, we solve the following local spectral problem in $\omega_{i}$

$$
A^{i, \text { snap }} \psi_{k}^{i, \text { snap }}=\lambda_{k} S^{i, \text { snap }} \psi_{k}^{i, \text { snap }}
$$


where

$$
A^{i \text {,snap }}=\left[a_{m n}^{\mathrm{snap}}\right]=a_{i}\left(\phi_{m}^{i}, \phi_{n}^{i}\right)=R_{i, \text { snap }} A^{i} R_{i, \text { snap }}^{T} \quad S^{i, \text { snap }}=\left[m_{m n}^{\text {snap }}\right]=s_{i}\left(\phi_{m}^{i}, \phi_{n}^{i}\right)=R_{i, \text { snap }} S^{i} R_{i, \text { snap }}^{T}
$$

and

$$
a_{i}(q, v)=\int_{E_{i}}\left(q \cdot n_{i}\right)\left(v \cdot n_{i}\right) d s, \quad s_{i}(q, v)=\int_{\omega_{i}} q v d x+\int_{\omega_{i}} \div q \div v d x
$$

To form a multiscale space, we arrange the eigenvalues in increasing order, and choose the first $M_{i}$ eigenvalues and take the corresponding eigenvectors $\psi_{k}^{i}=R_{i \text {,snap }} \psi_{k}^{i, \text { snap }}$ as the basis functions $\left(k=1, \ldots, M_{i}\right)$

$$
V^{q, H}=\operatorname{span}\left\{\psi_{k}^{i}: 1 \leq i \leq N_{e}, 1 \leq k \leq M_{i}\right\}, \quad R_{q}=\left[\psi_{1}^{1}, \ldots, \psi_{M_{1}}^{1}, \ldots, \psi_{1}^{N_{e}}, \ldots, \psi_{M_{N_{e}}}^{N_{e}}\right] .
$$

This space will be used as the approximation space for the flux.

\section{Flow Problem}

For the approximation of the flow problem on the fine grid, we use a Discontinuous Galerkin method [33-38]. Let $\mathcal{T}^{h}$ be a fine-grid partition of the domain $\Omega, \mathcal{E}^{h}$ be the set of facets in $\mathcal{T}^{h}$ and $\mathcal{E}^{h}=\mathcal{E}_{\text {int }}^{h} \cup \mathcal{E}_{\text {out }}^{h}\left(\mathcal{E}_{\text {int }}^{h}\right.$ is the set of interior facets and $\mathcal{E}_{\text {out }}^{h}$ is the set of boundary facets). We use the notations $K$ and $E$ to denote cell and facet in the fine grid $\mathcal{T}^{h}$. We define the jump $[u]$ and the average $\{u\}$ of a function $u$ on interior facet

$$
[u]_{E}=\left.u\right|_{K^{+}}-\left.u\right|_{K^{-}}, \quad\{u\}_{E}=\frac{\left.u\right|_{K^{+}}+\left.u\right|_{K^{-}}}{2},
$$

where $n$ is the unit normal vector on $E, K^{+}$and $K^{-}$are the two cells sharing the facet $E$. For $E \in \mathcal{E}_{\text {out }}^{h}$, we define

$$
[u]_{E}=\left.u\right|_{E}, \quad\{u\}_{E}=\left.u\right|_{E} .
$$

We have the following variational formulations for the DG approach: find $\left(u^{k+1}, p^{n+1}\right) \in V^{u} \times Q^{p}$ such that

$$
\begin{aligned}
a_{\mathrm{DG}}\left(u^{k+1}, v\right)+b_{\mathrm{DG}}\left(p^{k+1}, v\right) & =f_{u}(v), \quad \forall v \in V^{u}, \\
b_{\mathrm{DG}}\left(u^{k+1}, r\right) & =0, \quad \forall r \in Q^{p}
\end{aligned}
$$

where

$$
\begin{gathered}
a_{\mathrm{DG}}(u, v)=\int_{\Omega} R_{e}^{-1} \nabla u \cdot \nabla v d x-\sum_{E \in \mathcal{E}^{h}} \int_{E}\left(\left\{R_{e}^{-1} \nabla u \cdot n\right\} \cdot[v]+\left\{R_{e}^{-1} \nabla v \cdot n\right\} \cdot[u]-\int_{E} \frac{\gamma}{h} R_{e}^{-1}[u] \cdot[v]\right) d s, \\
b_{\mathrm{DG}}(u, p)=-\sum_{K \in \mathcal{T}^{H}} \int_{K} p \nabla u d x+\sum_{E \in \mathcal{E}^{h}} \int_{E} p[u] \cdot n d s, \quad f_{u}(v)=-\int_{\Omega}\left(S_{c} D^{-1} B^{k+1} q^{k+1}\right) \cdot v d x .
\end{gathered}
$$

The fine scale velocity space $V^{u}=\left\{v \in L^{2}(\Omega):\left.v\right|_{K} \in\left(\mathbb{P}_{1}(T)\right)^{2}, \forall K \in \mathcal{T}^{h}\right\}$ contains functions that are piecewise linear in each fine-grid element $K$ and are continuous along the fine-grid edges, but are discontinuous across coarse grid edges. For the pressure, we use the space of piecewise constant functions.

In the matrix form, we have

$$
\left(\begin{array}{cc}
A & G^{T} \\
G & 0
\end{array}\right)\left(\begin{array}{c}
u^{k+1} \\
p^{k+1}
\end{array}\right)=\left(\begin{array}{c}
F_{u}^{k+1} \\
0
\end{array}\right)
$$

where $F_{u}^{k+1}=F_{u}\left(B^{k+1}, q^{k+1}\right)$. 
Next, we present a multiscale method for solutions of the Stokes problem. Similarly, to the Mixed GMsFEM to obtain multiscale basis functions, we solve problems in local domains with various boundary conditions to form a snapshot space and use a spectral problem to perform a dimension reduction.

\section{Multiscale Approximation}

Next, we present the construction of the multiscale space for the coarse scale approximation of velocity. Note that, in the DG multiscale method we construct a multiscale basis in each coarse block [26,27,39-41]. Let $\mathcal{T}^{H}$ be a coarse-grid partition of the domain $\Omega$ with mesh size $H$ and $\mathcal{E}^{H}$ be the set of facets in $\mathcal{T}^{H}, \mathcal{E}^{H}=\mathcal{E}_{\text {int }}^{H} \cup \mathcal{E}_{\text {out }}^{H}$. For the pressure approximation, we use the piecewise constant function space $Q^{p, H}$ over the coarse cells. We define $V^{u, H}$ as the multiscale velocity space, which contains a set of basis functions supported in each coarse block $K$ (see Figure 3).

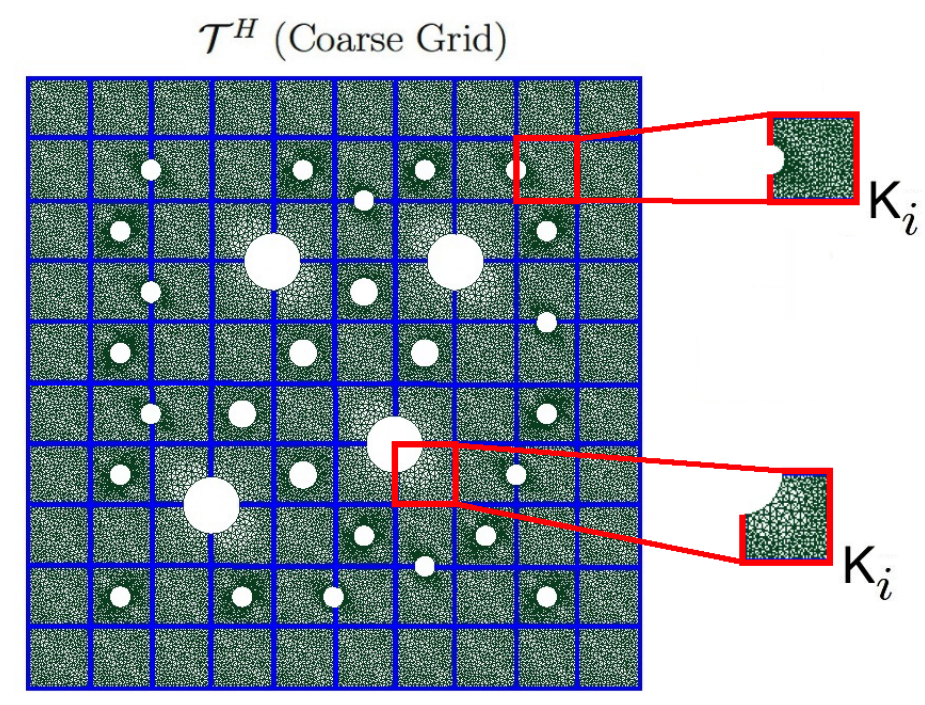

Figure 3. Illustration of a coarse grid and local domain $\left(K_{i}\right)$ for the Flow problem.

We construct a multiscale space for the velocity $\mathcal{T}^{H}$

$$
V^{u, H}=\operatorname{span}\left\{\psi_{i}\right\}_{i=1}^{N_{u}}, \quad Q^{p, H}=\left\{r \in L^{2}(\Omega):\left.r\right|_{K} \in P^{0}(K), \forall K \in \mathcal{T}_{H}\right\}
$$

and for the pressure, we use the space of piecewise constant functions over the coarse triangulation, $N_{u}=\operatorname{dim}\left(V^{u, H}\right)$ is the number of basis functions and $N_{p}=\operatorname{dim}\left(Q^{p, H}\right)$ is equal to the number of coarse grid cells.

For the coarse grid approximation, we use a DG approach and have the following variational formulation: find $\left(u_{H}^{k+1}, p_{H}^{k+1}\right) \in V^{u, H} \times Q^{p, H}$ such that

$$
\begin{aligned}
a_{\mathrm{DG}}\left(u_{H}^{k+1}, v\right)+g_{\mathrm{DG}}\left(p_{H}^{k+1}, v\right) & =f(v) \quad \forall v \in V^{u, H}, \\
g\left(u_{H}^{k+1}, r\right) & =0, \quad \forall r \in Q^{p, H},
\end{aligned}
$$

We can write the system in matrix form for each nonlinear iteration

$$
\left(\begin{array}{cc}
A_{H} & G_{H} \\
G_{H}^{T} & 0
\end{array}\right)\left(\begin{array}{l}
u_{H} \\
p_{H}
\end{array}\right)=\left(\begin{array}{c}
F_{H} \\
0
\end{array}\right),
$$

where

$$
A_{H}=R_{u} A R_{u}^{T}, \quad G_{H}=R_{u} G R_{p}^{T}, \quad F_{H}=R_{u} F_{u}
$$


Here $R_{u}$ and $R_{p}$ are the projection matrices

$$
R_{u}=\left[\psi_{1}, \ldots, \psi_{N_{u}}\right], \quad R_{p}=\left[\eta_{1}, \ldots, \eta_{N_{p}}\right],
$$

where $\left\{\psi_{i}\right\}_{i=1}^{N_{u}}$ are the multiscale basis functions for velocity.

For construction of the multiscale space for the velocity, we start with the construction of the snapshot space that contains an extensive set of basis functions formed by the solution of local problems with all possible boundary conditions up to the fine grid resolution. After that, we solve a spectral problem to select dominant modes of the snapshot space.

Snapshot space. We construct local snapshot basis in each coarse block $K_{i},(i=1, \cdots, N)$, where $N$ is the number of coarse blocks in $\Omega$. The local snapshot space consists of functions which are solutions $\phi_{l}^{i} \in V^{u}\left(K_{i}\right)$ of

$$
\begin{array}{rlrl}
-R_{e}^{-1} \Delta \phi_{l}^{i}+\nabla \eta & =0, & & x \in K_{i}, \\
\nabla \cdot \phi_{l}^{i} & =c, \quad x \in K_{i},
\end{array}
$$

with $\phi_{l}^{i}=\delta_{i}^{l}$ on $\partial K_{i}\left(l=1, \cdots, J_{i}\right)$, where $J_{i}$ is the number of fine grid nodes on the boundary of $K_{i}$, and $\delta_{i}^{l}$ is the discrete delta function defined on $\partial K_{i}$. This problem is solved on the fine mesh using some appropriate approximation spaces (DG). Here constant $c$ is chosen by the compatibility condition, $c=\frac{1}{\left|K_{i}\right|} \int_{\partial K_{i}} \delta_{i}^{l} \cdot n d s$.

Using local solutions, we form a local snapshot space in $K_{i}$

$$
V^{i, \text { snap }}=\left\{\phi_{l}^{i}: 1 \leq l \leq J_{i}\right\}, \quad R_{i, \text { snap }}=\left[\phi_{1}^{i}, \ldots, \phi_{J_{i}}^{i}\right] .
$$

Multiscale space. To reduce the size of the snapshot space, we solve the following local spectral problem in the snapshot space for $K_{i}$

$$
A^{i, \text { snap }} \psi_{k}^{i, \text { snap }}=\lambda_{k} S^{i, \text { snap }} \psi_{k}^{i, \text { snap }}
$$

where

$$
A^{i, \text { snap }}=R_{i, \text { snap }} A^{i} R_{i, \text { snap }}^{T} \quad S^{i \text { snap }}=R_{i, \text { snap }} S^{i} R_{i, \text { snap }}^{T}
$$

and $A^{i}$ is the matrix representation of the bilinear form $a_{i}(u, v)$ and $S^{i}$ is the matrix representation of the bilinear form $s_{i}(u, v)$

$$
a_{i}(u, v)=\int_{K_{i}} R_{e}^{-1} \nabla u \cdot \nabla v d x, \quad s_{i}(u, v)=\int_{\partial K_{i}} u \cdot v d x .
$$

We remark that the integral in $s_{i}(u, v)$ is defined on the boundary of the coarse block. In this case, the number of the spectral problem equals the number of coarse blocks.

We arrange the eigenvalues in increasing order and choose the first eigenvectors corresponding to the first $M_{i}$ the smallest eigenvalues $\psi_{k}^{i}=R_{i, \text { snap }} \psi_{k}^{i, \text { snap }}$ as the basis functions $\left(k=1, \ldots, M_{i}\right)$

$$
V_{i}^{u, H}=\operatorname{span}\left\{\psi_{k}^{i}: 1 \leq i \leq N_{e}, 1 \leq k \leq M_{i}\right\}, \quad R_{u}=\left[\psi_{1}^{1}, \ldots, \psi_{M_{1}}^{1}, \ldots, \psi_{1}^{N_{c}}, \ldots, \psi_{M_{N_{c}}}^{N_{c}}\right] .
$$

This space will be used as the approximation space for the velocity. The global multiscale space $V^{u, H}$ is the combination of the local ones, i.e.,

$$
V^{u, H}=\operatorname{span}\left\{\phi_{k}^{i}: 1 \leq k \leq L_{i}, 1 \leq i \leq N\right\} .
$$

\section{Numerical Results}

We present numerical results for the model problem in the perforated domain. We use the Generalized Multiscale Finite Element method (GMsFEM) to construct a coarse grid approximation. 
The presented method based on the calculations of local multiscale basis functions in order to form a projection matrix that is used to construct a lower-dimensional approximation. After obtaining the solution of the reduced-order system, we reconstruct a fine grid resolution using a transposed projection matrix. For error calculations, we use a solution of the problem with traditional finite element approximation on the fine grid as the reference solution.

A computational perforated domain is presented in Figure 4 and has dimensions $\Omega=[0,1] \times[0,1]$. For the approximation of the perforated domain, we use an unstructured fine grid with triangular cells that resolves perforations on the grid level. This can lead to the large discrete system of equations for the traditional finite element approximations. To reduce the size of the system, we present a multiscale solver that used to construct a reduced-order approximation on the coarse grid. The coarse grid can be either structured or unstructured. In this work, for simplicity, we consider a $10 \times 10$ structured grid to illustrate the multiscale method. In many applications, it is easier to use structured coarse grids and resolve the subgrid effects as we do in our simulations. We construct an unstructured fine mesh that contains 61,912 cells, 93,668 facets and 31,727 vertices. The coarse grid is uniform, which contains 100 cells, 220 facets and 121 vertices. We set $R_{e}^{-1}=1, D=10, S_{c}=1$ and $g=1$.
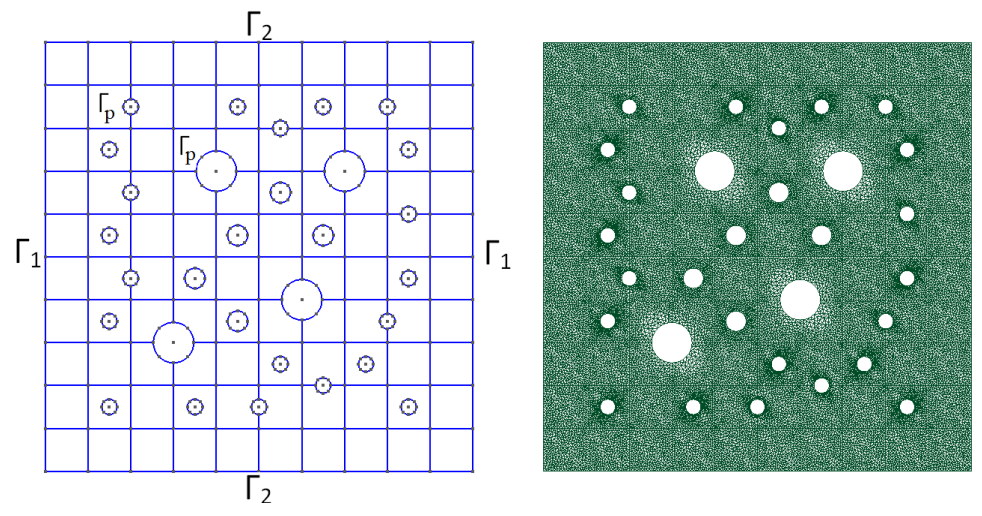

Figure 4. Perforated domain with coarse (left) and fine grids (right).

In Figures 5-7, we present the results of the proposed multiscale method. The distribution of the magnitude fields ( $B$ and $q$ ) are presented in Figures 5 and 6 . In Figure 7 , we show the velocity field. At the top of the figures, we presented the fine-scale solutions. Multiscale solutions are present on the bottom figure with $D O F_{c}=980$ for $q$ and $D O F_{c}=4100$ for velocity field $u$. Note that, we use the piecewise constant functions for multiscale solution of $B$. We use a Picard iteration and perform three iterations for all cases with $\epsilon_{B}=10^{-9}$.
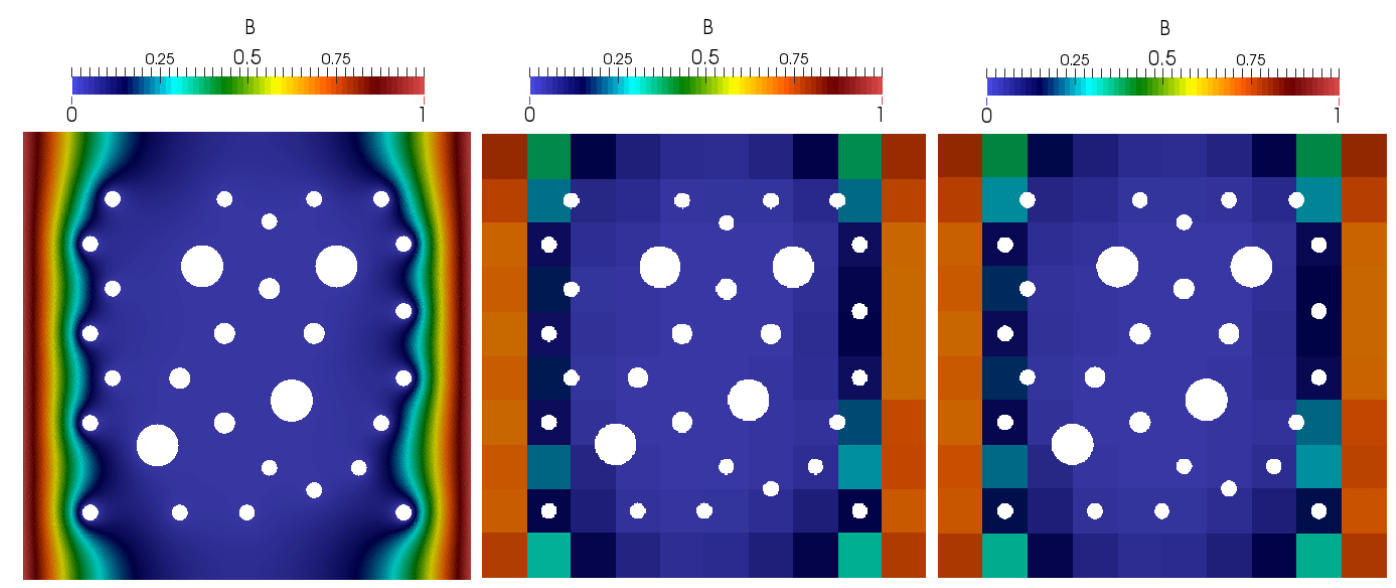

Figure 5. The distribution of the magnetic field. (Left): Reference (fine grid) solution, $D O F_{f}=155,580$. (Center): Cell average for reference (fine grid) solution. (Right): Multiscale solution, $D O F_{\mathcal{C}}=980$ $\left(0.48 \%\right.$ from $\left.D O F_{f}\right)$. 

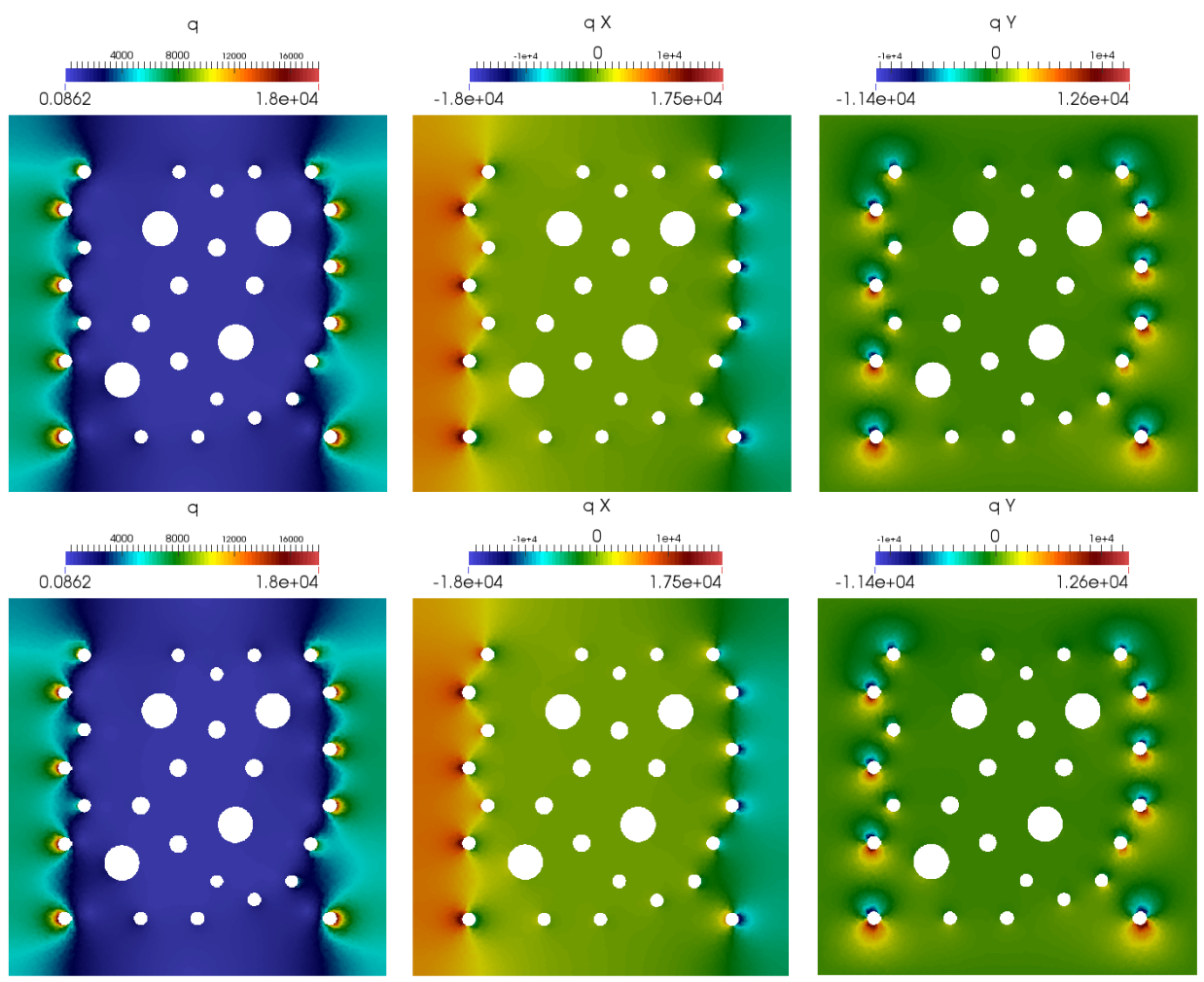

Figure 6. The distribution of the flux. Magnitude of flux, $\mathrm{x}$ and y components of flux, $q, q_{x}, q_{y}$ (from left to right). (Top): Reference (fine grid) solution, $D O F_{f}=155,580$. (Bottom): Multiscale solution, $D O F_{c}=980\left(0.48 \%\right.$ from $\left.D O F_{f}\right)$.
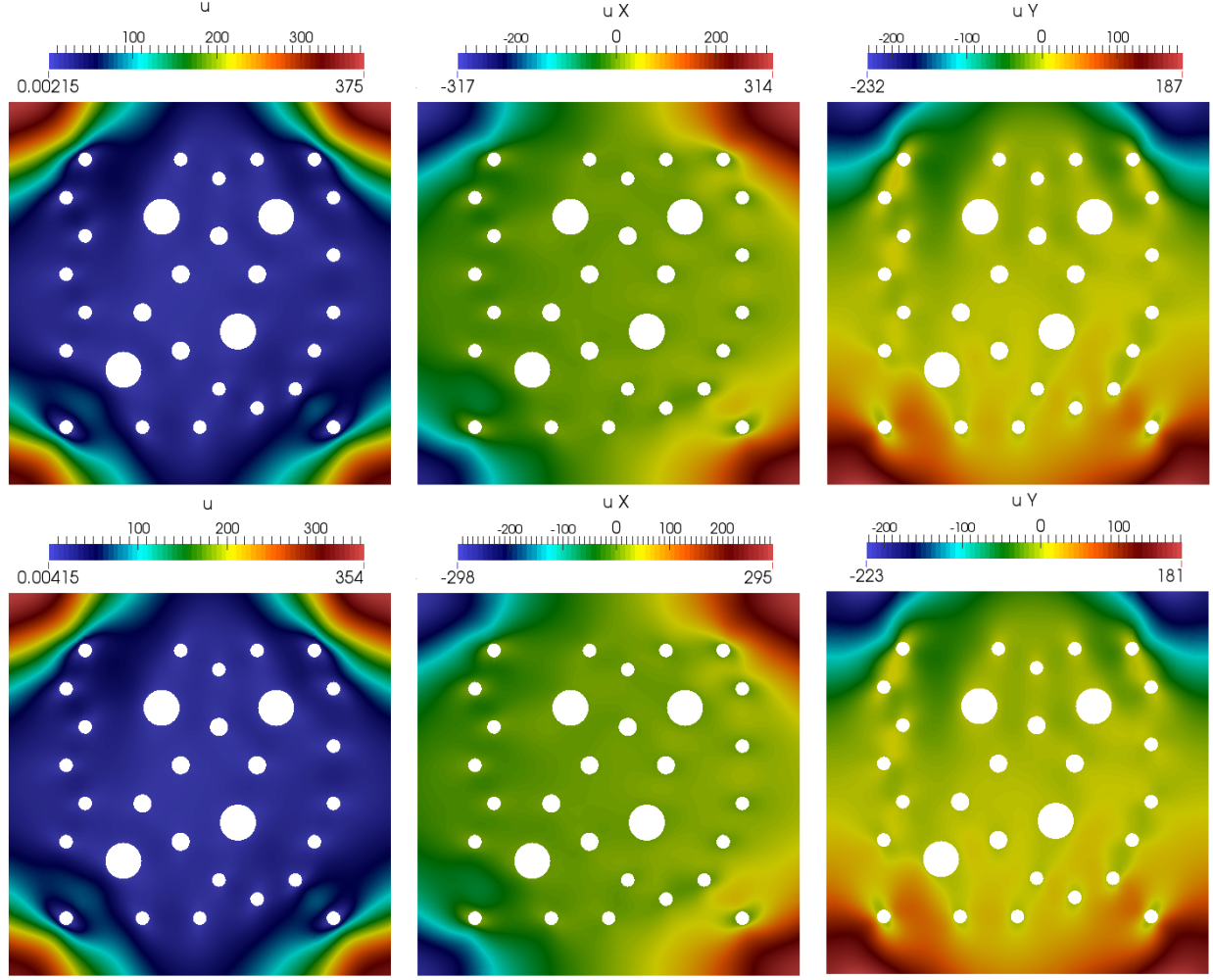

Figure 7. The distribution of the velocity. Magnitude of velocity, $\mathrm{x}$ and $\mathrm{y}$ components of velocity, $u$, $u_{x} u_{y}$ (from left to right). (Top): Reference (fine grid) solution, $D O F_{f}=433,384$. (Bottom): Multiscale solution, $D O F_{c}=4100\left(0.9 \%\right.$ from $\left.D O F_{f}\right)$. 
We calculate following errors:

$$
\begin{aligned}
e^{u} & =\sqrt{\frac{\int_{\Omega}\left|u-u_{m s}\right|^{2} d x}{\int_{\Omega} u^{2} d x}} \cdot 100 \%, \quad e^{q}=\sqrt{\frac{\int_{\Omega}\left|q-q_{m s}\right|^{2} d x}{\int_{\Omega} q^{2} d x}} \cdot 100 \%, \\
e_{L_{2}}^{\bar{p}} & =\sqrt{\frac{\int_{\Omega}\left|\bar{p}-p_{H}\right|^{2} d x}{\int_{\Omega} \bar{p}^{2} d x}} \cdot 100 \%, \quad e_{L_{2}}^{\bar{B}}=\sqrt{\frac{\int_{\Omega}\left|\bar{B}-B_{H}\right|^{2} d x}{\int_{\Omega} \bar{B}^{2} d x}} \cdot 100 \%,
\end{aligned}
$$

where $(q, B)$ and $(u, p)$ are the reference solutions using traditional fine grid solvers, $\bar{B}$ and $\bar{p}$ are the coarse cell average for reference solutions, $\left(q_{H}, B_{H}\right)$ and $\left(u_{H}, p_{H}\right)$ are the coarse grid solutions using presented multiscale solvers, $q_{m s}=R_{q}^{T} q_{H}$ and $u_{m s}=R_{u}^{T} u_{H}$ are the reconstructed multiscale solutions.

In Table 1 , we show the relative error for $q$ and $B$. We presented errors for different velocities: (1) multiscale velocities with $D O F_{c}=1100$ (10 basis), (2) multiscale velocities with $D O F_{c}=2100$ (20 basis), (3) multiscale velocities with $D O F_{c}=3100$ (30 basis), (4) multiscale velocities with $D O F_{c}=4100$ (40 basis), (5) multiscale velocities with $D O F_{c}=6100$ (60 basis) and (6) fine-scale solution with $D O F_{f}=433,384$. We see the relative error for $q$ is reduced from $4.1 \%$ to $0.8 \%$, and the coarse cell average error for $B$ is around one percent.

Table 1. Relative errors for flux and magnetic field with a different number of multiscale basis functions

\begin{tabular}{|c|c|c|}
\hline $\mathrm{DOF}_{\mathrm{c}}$ & Flux, $e^{q}(\%)$ & Magnetic Field, $e^{\bar{B}}(\%)$ \\
\hline \multicolumn{3}{|c|}{ u-multiscale solution with $D O F_{c}=1100$} \\
\hline 320 & 4.170 & 1.690 \\
\hline 540 & 1.181 & 1.578 \\
\hline 760 & 0.958 & 1.578 \\
\hline 980 & 0.835 & 1.578 \\
\hline \multicolumn{3}{|c|}{ u-multiscale solution with $D O F_{c}=2100$} \\
\hline 320 & 4.165 & 1.688 \\
\hline 540 & 1.176 & 1.577 \\
\hline 760 & 0.953 & 1.577 \\
\hline 980 & 0.831 & 1.577 \\
\hline \multicolumn{3}{|c|}{ u-multiscale solution with $D O F_{c}=3100$} \\
\hline 320 & 4.164 & 1.688 \\
\hline 540 & 1.176 & 1.577 \\
\hline 760 & 0.953 & 1.577 \\
\hline 980 & 0.830 & 1.577 \\
\hline \multicolumn{3}{|c|}{ u-multiscale solution with $D O F_{c}=4100$} \\
\hline 320 & 4.164 & 1.688 \\
\hline 540 & 1.176 & 1.577 \\
\hline 760 & 0.952 & 1.577 \\
\hline 980 & 0.830 & 1.577 \\
\hline \multicolumn{3}{|c|}{ u-multiscale solution with $D O F_{c}=6100$} \\
\hline 320 & 4.163 & 1.688 \\
\hline 540 & 1.176 & 1.577 \\
\hline 760 & 0.952 & 1.577 \\
\hline 980 & 0.830 & 1.577 \\
\hline \multicolumn{3}{|c|}{ u-fine-scale solution with $D O F_{f}=433,384$} \\
\hline 320 & 4.161 & 1.688 \\
\hline 540 & 1.174 & 1.577 \\
\hline 760 & 0.954 & 1.577 \\
\hline 980 & 0.832 & 1.577 \\
\hline
\end{tabular}
for $10 \times 10$ meshes. $D O F_{f}=155,580$. 
In Table 2 we present the relative errors for velocity and for pressure field. We presented errors for $u$ and $p$ with different $B$ : (1) multiscale magnetic field with $D O F_{c}=320$ (1 basis), (2) multiscale magnetic field with $D O F_{c}=540$ (2 basis), (3) multiscale magnetic field with $D O F_{c}=760$ ( 3 basis), (4) multiscale magnetic field with $D O F_{c}=980$ (4 basis) and (5) fine-scale solution with $D O F_{f}=155,580$. We see the relative velocity error reduces from $60 \%$ to $8 \%$ with a larger number of multiscale basis functions. We use a discontinuous Galerkin Generalized Multiscale Finite Element Method (DG-GMsFEM) for the flow problem and Mixed Generalized Multiscale Finite Element Method (Mixed GMsFEM) for the magnetic field problem. The construction of basis functions in both methods follows the general framework of GMsFEM by using local snapshots and local spectral problems. The errors can vary depending on a choice of discretization. In order to further error reduction for the multiscale approximation of the flow problem, we will apply oversampling techniques in future work [39].

Table 2. Relative errors for velocity and pressure with different number of multiscale basis functions for $10 \times 10$ meshes. $D O F_{f}=433,384$.

\begin{tabular}{|c|c|c|}
\hline$D^{\prime} F_{c}$ & Velocity, $e^{u}(\%)$ & Pressure, $e^{\bar{p}}(\%)$ \\
\hline \multicolumn{3}{|c|}{ B-multiscale solution with $D O F_{c}=320$} \\
\hline 1100 & 60.501 & 25.800 \\
\hline 2100 & 16.689 & 2.005 \\
\hline 3100 & 11.281 & 1.226 \\
\hline 4100 & 9.792 & 1.138 \\
\hline 6100 & 8.843 & 1.078 \\
\hline \multicolumn{3}{|c|}{ B-multiscale solution with $D O F_{c}=540$} \\
\hline 1100 & 60.477 & 25.798 \\
\hline 2100 & 16.694 & 1.996 \\
\hline 3100 & 11.282 & 1.225 \\
\hline 4100 & 9.792 & 1.138 \\
\hline 6100 & 8.843 & 1.078 \\
\hline \multicolumn{3}{|c|}{ B-multiscale solution with $D O F_{c}=760$} \\
\hline 1100 & 60.473 & 25.797 \\
\hline 2100 & 16.695 & 1.997 \\
\hline 3100 & 11.282 & 1.225 \\
\hline 4100 & 9.792 & 1.138 \\
\hline 6100 & 8.843 & 1.078 \\
\hline \multicolumn{3}{|c|}{ B-multiscale solution with $D O F_{c}=980$} \\
\hline 1100 & 60.472 & 25.797 \\
\hline 2100 & 16.694 & 1.997 \\
\hline 3100 & 11.282 & 1.225 \\
\hline 4100 & 9.792 & 1.138 \\
\hline 6100 & 8.843 & 1.078 \\
\hline \multicolumn{3}{|c|}{ B-fine-scale solution with $D O F_{f}=155,580$} \\
\hline 1100 & 59.420 & 25.300 \\
\hline 2100 & 16.615 & 1.878 \\
\hline 3100 & 11.238 & 1.199 \\
\hline 4100 & 9.754 & 1.112 \\
\hline 6100 & 8.809 & 1.055 \\
\hline
\end{tabular}

\section{Conclusions}

We considered the magnetohydrodynamics (MHD) problem in a perforated domain. A mathematical model was described using a coupled system of equations for the magnetic field and for the flow problem. We constructed a fine grid that resolved perforations on the grid level in order to use a traditional approximation. For the solution on the fine grid, we constructed approximation using 
the mixed finite element method. To reduce the size of the fine grid system with an accurate solution on the coarse grid, a Mixed Generalized Multiscale Finite Element Method (Mixed GMsFEM) was presented. Numerical results were presented for a two-dimensional model problem in a perforated domain and we studied the influence of the number of multiscale basis functions on the method accuracy. In the multiscale solver, the main computational gain was due to fewer multiscale basis functions, which are used to reduce the size of the system. For the traditional fine grid solutions, we have $D O F_{f}=155,580$ for the magnetic field problem and $D O F_{f}=433,384$ for the flow problem. For multiscale solvers, we have $0.9 \%$ error for $q$ with three multiscale basis functions with $D O F_{c}=760$ $\left(0.48 \%\right.$ of $\left.D O F_{f}\right)$ and $9 \%$ error for $u$ with 40 multiscale basis functions with $D O F_{c}=4100(0.9 \%$ of $D O F_{f}$ ). In the future, we plan to consider oversampling techniques and construct a multiscale solver for three-dimensional problems.

Author Contributions: Writing-original draft, M.V.; Writing—review \& editing, V.A., Q.T., E.T.C. and Y.E. All authors have read and agreed to the published version of the manuscript.

Funding: V.A.'s work is supported by the mega-grant of the Russian Federation Government N14.Y26.31.0013 and RFBR N19-31-90076. M.V.'s work is supported by the mega-grant of the Russian Federation Government N14.Y26.31.0013. The research of Qili Tang is partially supported by the National Natural Science Foundation of China (No:r 11701151), the Natural Science Foundation of Hunan Province (No: 2019JJ40279), Excellent Youth Program of Scientific Research Project of Hunan Provincial Department of Education (No: 18B064) and International Scientific and Technological Innovation Cooperation Base of Hunan Province for Computational Science (No: 2018WK4006). The research of Eric Chung is partially supported by the Hong Kong RGC General Research Fund (Project numbers 14304217 and 14302018) and CUHK Faculty of Science Direct Grant 2018-19. Y.E. would like to thank the partial support from NSF 1620318 and 1934904. Y.E. would also like to acknowledge the support of Mega-grant of the Russian Federation Government (N 14.Y26.31.0013).

Conflicts of Interest: The authors declare no conflict of interest.

\section{References}

1. Moreau, R.J. Magnetohydrodynamics; Kluwer Academic Publishers: Dordrecht, The Netherlands, 2013; Volume 3.

2. Davidson, P.A. An Introduction to Magnetohydrodynamics. Am. J. Phys. 2002, 70, 781. [CrossRef]

3. Gerbeau, J.F.; Le Bris, C.; Lelièvre, T. Mathematical Methods for the Magnetohydrodynamics of Liquid Metals; Clarendon Press: London, UK, 2006.

4. Dong, X.; He, Y.; Zhang, Y. Convergence analysis of three finite element iterative methods for the 2D/3D stationary incompressible magnetohydrodynamics. Comput. Methods Appl. Mech. Eng. 2014, 276, $287-311$. [CrossRef]

5. Dong, X.; He, Y. Convergence of some finite element iterative methods related to different Reynolds numbers for the 2D/3D stationary incompressible magnetohydrodynamics. Sci. China Math. 2016, 59, 589-608. [CrossRef]

6. Dong, X.; He, Y. The Oseen Type Finite Element Iterative Method for the Stationary Incompressible Magnetohydrodynamics. Adv. Appl. Math. Mech. 2017, 9, 775-794. [CrossRef]

7. Dong, X.; He, Y.; Wei, H.; Zhang, Y. Local and parallel finite element algorithm based on the partition of unity method for the incompressible MHD flow. Adv. Comput. Math. 2018, 44, 1295-1319. [CrossRef]

8. Tang, Q.; Huang, Y. Local and parallel finite element algorithm based on Oseen-type iteration for the stationary incompressible MHD flow. J. Sci. Comput. 2017, 70, 149-174. [CrossRef]

9. Tang, Q.; Huang, Y. Analysis of Local and Parallel Algorithm for Incompressible Magnetohydrodynamics Flows by Finite Element Iterative Method. Commun. Comput. Phys. 2019, 25, 729-751. [CrossRef]

10. Badia, S.; Codina, R.; Planas, R. On an unconditionally convergent stabilized finite element approximation of resistive magnetohydrodynamics. J. Comput. Phys. 2013, 234, 399-416. [CrossRef]

11. Greif, C.; Li, D.; Schötzau, D.; Wei, X. A mixed finite element method with exactly divergence-free velocities for incompressible magnetohydrodynamics. Comput. Methods Appl. Mech. Eng. 2010, 199, $2840-2855$. [CrossRef]

12. Hiptmair, R.; Li, L.; Mao, S.; Zheng, W. A fully divergence-free finite element method for magnetohydrodynamic equations. Math. Models Methods Appl. Sci. 2018, 28, 659-695. [CrossRef] 
13. Hu, K.; Ma, Y.; Xu, J. Stable finite element methods preserving $\nabla \cdot\{B\}=0$ exactly for MHD models. Numer. Math. 2017, 135, 371-396. [CrossRef]

14. Jikov, V.V.; Kozlov, S.M.; Oleinik, O.A. Homogenization of Differential Operators and Integral Functionals; Springer: Berlin, Germany, 1991.

15. Bakhvalov, N.; Panasenko, G. Homogenization in Periodic Media, Mathematical Problems of the Mechanics of Composite Materials; Nauka: Moscow, Russia, 1984.

16. Allaire, G.; Brizzi, R. A multiscale finite element method for numerical homogenization. SIAM J. Multiscale Modeling Simul. 2005, 4, 790-812. [CrossRef]

17. Efendiev, Y.; Hou, T. Multiscale Finite Element Methods: Theory and Applications. In Surveys and Tutorials in the Applied Mathematical Sciences; Springer: New York, NY, USA, 2009; Volume 4.

18. Efendiev, Y.; Galvis, J.; Hou, T.Y. Generalized multiscale finite element methods (GMsFEM). J. Comput. Phys. 2013, 251, 116-135. [CrossRef]

19. Ohlberger, M.; Schindler, F. Error control for the localized reduced basis multi-scale method with adaptive on-line enrichment. arXiv 2015, arXiv:1501.05202.

20. Muljadi, B.; Narski, J.; Lozinski, A.; Degond, P. Non-conforming multiscale finite element method for Stokes flows in heterogeneous media. Part I: Methodologies and numerical experiments. arXiv 2014, arXiv:1404.2837.

21. Le Bris, L.; Legoll, F.; Lozinski, A. An MsFEM type approach for perforated domains. Multiscale Model. Simul. 2014, 12, 1046-1077. [CrossRef]

22. Henning, P.; Ohlberger, M. The heterogeneous multiscale finite element method for elliptic homogenization problems in perforated domains. Numer. Math. 2009, 113, 601-629. [CrossRef]

23. Chen, Z.; Hou, T. A mixed multiscale finite element method for elliptic problems with oscillating coefficients. Math. Comput. 2002, 72, 541-576. [CrossRef]

24. Brown, D.L.; Peterseim, D. A multiscale method for porous microstructures. arXiv 2014, arXiv:1411.1944.

25. Efendiev, Y.; Galvis, J.; Wu, X.H. Multiscale finite element methods for high-contrast problems using local spectral basis functions. J. Comput. Phys. 2011, 230, 937-955. [CrossRef]

26. Chung, E.T.; Efendiev, Y.; Li, G.; Vasilyeva, M. Generalized multiscale finite element methods for problems in perforated heterogeneous domains. Appl. Analys. 2016, 95, 2254-2279. [CrossRef]

27. Chung, E.T.; Efendiev, Y.; Vasilyeva, M.; Wang, Y. A multiscale discontinuous Galerkin method in perforated domains. In Proceedings of the Institute of Mathematics and Mechanics, Maringá, Brazil, 22-23 September 2016; Volume 42, pp. 212-229.

28. Chung, E.T.; Efendiev, Y.; Lee, C.S. Mixed generalized multiscale finite element methods and applications. Multiscale Model. Simul. 2015, 13, 338-366. [CrossRef]

29. Chan, H.Y.; Chung, E.; Efendiev, Y. Adaptive mixed GMsFEM for flows in heterogeneous media. Numer. Math. Theory Methods Appl. 2016, 9, 497-527. [CrossRef]

30. Chung, E.T.; Leung, W.T.; Vasilyeva, M. Mixed GMsFEM for second order elliptic problem in perforated domains. J. Comput. Appl. Math. 2016, 304, 84-99. [CrossRef]

31. Chung, E.T.; Leung, W.T.; Vasilyeva, M.; Wang, Y. Multiscale model reduction for transport and flow problems in perforated domains. J. Comput. Appl. Math. 2018, 330, 519-535. [CrossRef]

32. Gunzburger, M.D.; Meir, A.J.; Peterson, J.S. On the existence, uniqueness, and finite element approximation of solutions of the equations of stationary, incompressible magnetohydrodynamics. Math. Comput. 1991, 56, 523-563. [CrossRef]

33. Arnold, D.; Brezzi, F.; Cockburn, B.; Marini, L. Unified analysis of discontinuous Galerkin methods for elliptic problems. SIAM J. Numer. Anal. 2002, 39, 1749-1779. [CrossRef]

34. Ewing, R.; Wang, J.; Yang, Y. A stabilized discontinuous finite element method for elliptic problems. Numer. Linear Algebra Appl. 2003, 10, 83-104. [CrossRef]

35. Lazarov, R.; Pasciak, J.; Schöberl, J.; Vassilevski, P. Almost optimal interior penalty discontinuous approximations of symmetric elliptic problems on non-matching grids. Numer. Math. 2003, 96, 295-315. [CrossRef]

36. Lazarov, R.; Tomov, S.; Vassilevski, P. Interior penalty discontinuous approximations of elliptic problems. Comput. Methods Appl. Math. 2001, 1, 367-382. [CrossRef]

37. Riviere, B. Discontinuous Galerkin Methods for Solving Elliptic And Parabolic Equations: Theory and Implementation; SIAM: Philadelphia, PA, USA, 2008. 
38. Girault, V.; Riviére, B.; Wheeler, M. A discontinuous Galerkin method with nonoverlapping domain decomposition for the Stokes and Navier-Stokes problems. Math. Comput. 2005, 74, 53-84. [CrossRef]

39. Chung, E.T.; Vasilyeva, M.; Wang, Y. A conservative local multiscale model reduction technique for Stokes flows in heterogeneous perforated domains. J. Comput. Appl. Math. 2017, 321, 389-405. [CrossRef]

40. Chung, E.T.; Efendiev, Y.; Leung, W.T. An adaptive generalized multiscale discontinuous Galerkin method for high-contrast flow problems. Multiscale Model. Simul. 2018, 16, 1227-1257. [CrossRef]

41. Chung, E.; Leung, W.T. A sub-grid structure enhanced discontinuous Galerkin method for multiscale diffusion and convection-diffusion problems. Commun. Comput. Phys. 2013, 14, 370-392. [CrossRef]

(C) 2020 by the authors. Licensee MDPI, Basel, Switzerland. This article is an open access article distributed under the terms and conditions of the Creative Commons Attribution (CC BY) license (http:/ / creativecommons.org/licenses/by/4.0/). 